C hiapas posee una de las más diversas floras de México (RamírezMarcial et al., 1998; Villaseñor, 2003) con alrededor de 8,250 especies de plantas (Breedlove, 1981). No obstante, el conocimiento de su riqueza florística es aún incompleto (MartínezMeléndez et al., 2008) y los intentos por cuantificarla con precisión se ven obstruidos por la existencia de un gran número de áreas poco exploradas o inaccesibles, principalmente aquellas que se encuentran fuera de reservas naturales y que han aportado recientemente, la descripción de nuevas especies (Ortega-Ortíz y Ortega-Ortíz, 1998; León y Martínez-Gordillo, 2008; Lozada-Pérez, 2008) y nuevas áreas de distribución (Contreras-Medina y Luna-Vega, 2001; Olvera-García y Martínez-S, 2002; López-Velázquez et al., 2007; Beutelspacher-Baigts y Moreno-Molina, 2010).

La zona sujeta a protección ecológica 'La Pera' ( $16^{\circ} 51^{\prime}$ ' 47.57' N y $93^{\circ}$ $19^{\prime} 34.85$ ' O) se encuentra ubicada en la parte central del municipio de Berriozábal, Chiapas, formando parte de acuerdo a Mullerried (1957), de la región fisiográfica Depresión Central. Dentro de la reserva, el bosque tropical perennifolio (Rzedowski, 2006) es la comunidad vegetal mejor representada y con mayor extensión, la cual se desarrolla sobre un terreno kárstico con numerosas grietas, simas y cavernas. En un esfuerzo por contribuir al conocimiento de este ecosistema en Chiapas, se está llevando a cabo un estudio que tiene como objetivo analizar la estructura y composición florística de las comunidades vegetales presentes en el municipio de Berriozábal y como parte de este proyecto se han realizado numerosas colectas botánicas, dando como resultado el primer registro para la flora de Chiapas de $P i$ lea pteridophylla y Ocotea heribertoi.

Pilea es el género más grande de la familia Urticaceae, comprende aproximadamente 715 especies (Monro,

\title{
Primer Registro de PILEA Pteridophylla A.K.Monro (Urticaceae) y Ocotea HERIBERTOI T.WENDT (LAURACEAE) PARA LA FLORA DE CHIAPAS
}

\author{
Andrés Ernesto Ortiz-Rodríguez ${ }^{1}, 4$, Francisco Lorea-Hernández ${ }^{1}$, \\ Miguel A. Pérez-Farrera² y Alexandre K. Monro ${ }^{3}$ \\ ${ }^{1}$ Instituto de Ecología, A.C. \\ ${ }^{2}$ Herbario Eizi Matuda (HEM) Facultad de Ciencias Biológicas, \\ Universidad de Ciencias y Artes de Chiapas \\ ${ }^{3}$ Department of Botany. The Natural History Museum London \\ ${ }^{4}$ Autor para la correspondencia: ortizrodriguez.ae@gmail.com
}

Resumen: Se registra por primera vez para el estado de Chiapas Pilea pteridophylla A.K.Monro y Ocotea heribertoi T.Wendt. Ambas especies de distribución restringida y endémicas de México, eran conocidas hasta el momento para los estados de Tabasco y Oaxaca respectivamente. Fueron encontradas en la zona sujeta a protección ecológica "La Pera" en el municipio de Berriozábal, Chiapas, México.

Palabras clave: Berriozábal, Chiapas, nuevo registro, Ocotea, Pilea, bosque tropical perennifolio

Abstract: Pilea pteridophylla A.K.Monro and Ocotea heribertoi T.Wendt are recorded for the first time for Chiapas. Both species are restricted in distribution and endemic to Mexico. Formerly these species have only been found in Tabasco and Oaxaca respectively. The two species were collected in "La Pera" an ecological protection zone in the municipality of Berriozábal, Chiapas, Mexico.

Keywords: Berriozábal, Chiapas, new record, Ocotea, Pilea, tropical rain forest

2004) en todo el mundo y se distribuye a lo largo de los trópicos, subtrópicos y regiones templadas (con la excepción de Australia, Monro, 2006). Se distingue fácilmente de otras urticáceas neotropicales por la combinación de hojas opuestas y estípulas intrapeciolares, así como por la ausencia de tricomas urticantes (Rodríguez y Monro, 2008). La mayoría de las especies son hierbas suculentas, epifitas o pequeños arbustos de flores unisexuales (raramente bisexuales) que crecen en sombra en altitudes entre 1,000 y 3,000 m s.n.m. (Monro, 2006).
Pilea pteridophylla (en Berriozábal, Chiapas), es una hierba de 40 a 90 cm; rupícola. Tallos erectos, obscuros cuando secos, glabros; cistolitos ausentes; entrenudos 5-20 × 1.5-2.5 mm; más o menos estriados. Hojas iguales en el mismo nudo; láminas 80-120 × 15-20 mm, linear-lanceoladas, pectinadas, cartáceas, venación pinnada, los nervios secundarios de 20-25 pares, débilmente curveados; haz y envés verde grisáceos cuando secos, glabros, con cistolitos en forma de "V","Y" y "X"; base simétrica, decurrente, atenuada; márgenes pectinados, la base 
de 1/8 a 1/6 entera; ápice débilmente cuspidado. Pecíolos iguales en el mismo nudo menos de $2 \mathrm{~mm}$, glabros. Inflorescencias paniculadas, 9-14 por tallo, unisexuales, pistiladas, 1 ó 2 por axila, pedúnculos y pedicelos subtendidos por brácteas estipuliformes; flores blancas.

Pilea pteridophylla es inconfundible, sus hojas se asemejan a las frondas de un helecho en color, textura, así como en forma. Es la única especie de Pilea con hojas pectinadas de América (Monro, 1999). Es endémica de México, conocida sólo de Teapa, Tabasco, de donde fue descrita originalmente (Monro, 1999). En Chiapas esta especie presenta una mayor longitud total, hojas más grandes, mayor distancia entre nudos y la condición pectinada de las hojas es menos marcada que en el ejemplar tipo (Figura 1). Esto sin duda, motiva a realizar proyectos de investigación con ambas localidades en un sentido genético, morfológico y geográfico.

Material examinado: zona sujeta a protección ecológica "La Pera", Berriozábal, Chiapas. Predio "Peña Flor" $16^{\circ} 51$ ' 50.6" N y 93 19' 51.7" O 1068 m s.n.m. Ortiz-Rodríguez Andrés Ernesto 320 (HEM).

El género Ocotea es de los grupos más diversos dentro de la familia Lauraceae, con alrededor de 350 especies, distribuidas principalmente en América tropical (de México a Argentina) aunque también se encuentran en Madagascar y en el sur de África (van der Werff, 1991 y 2002). El género, se caracteriza por la presencia de estambres con cuatro esporangios arreglados en dos pares más o menos verticales, glabros o casi así; los estaminodios del cuarto verticilo con filamento y ápice relativamente bien definidos (aunque a veces ausentes); flores unisexuales o hermafroditas; tépalos libres, erectos o extendidos; superficie interior de los tépalos glabra o pubescente, pero sin tricomas papilosos (o solo en el ápice), tépalos deciduos o raramente persistentes en el fruto (van der Werff, 2002).

Ocotea heribertoi (en Berriozábal, Chiapas) es un árbol de 18 metros de alto. Ramas estriadas, diminuta y escasamente adpreso pubescentes; yemas terminales blancas, adpreso pubescentes. Hojas de 8 a $13 \mathrm{~cm}$ de largo, firmemente cartáceas, elípticas a oblongoelípticas, alternas, venación pinnada, la base aguda o cuneada-decurrente, el margen plano, el ápice redondeado, glabras en ambas superficies, vena central, venas secundarias y terciarias inconspicuas o poco visibles en el haz; en el envés, la nervadura central prominente, venación secundaria y ter-

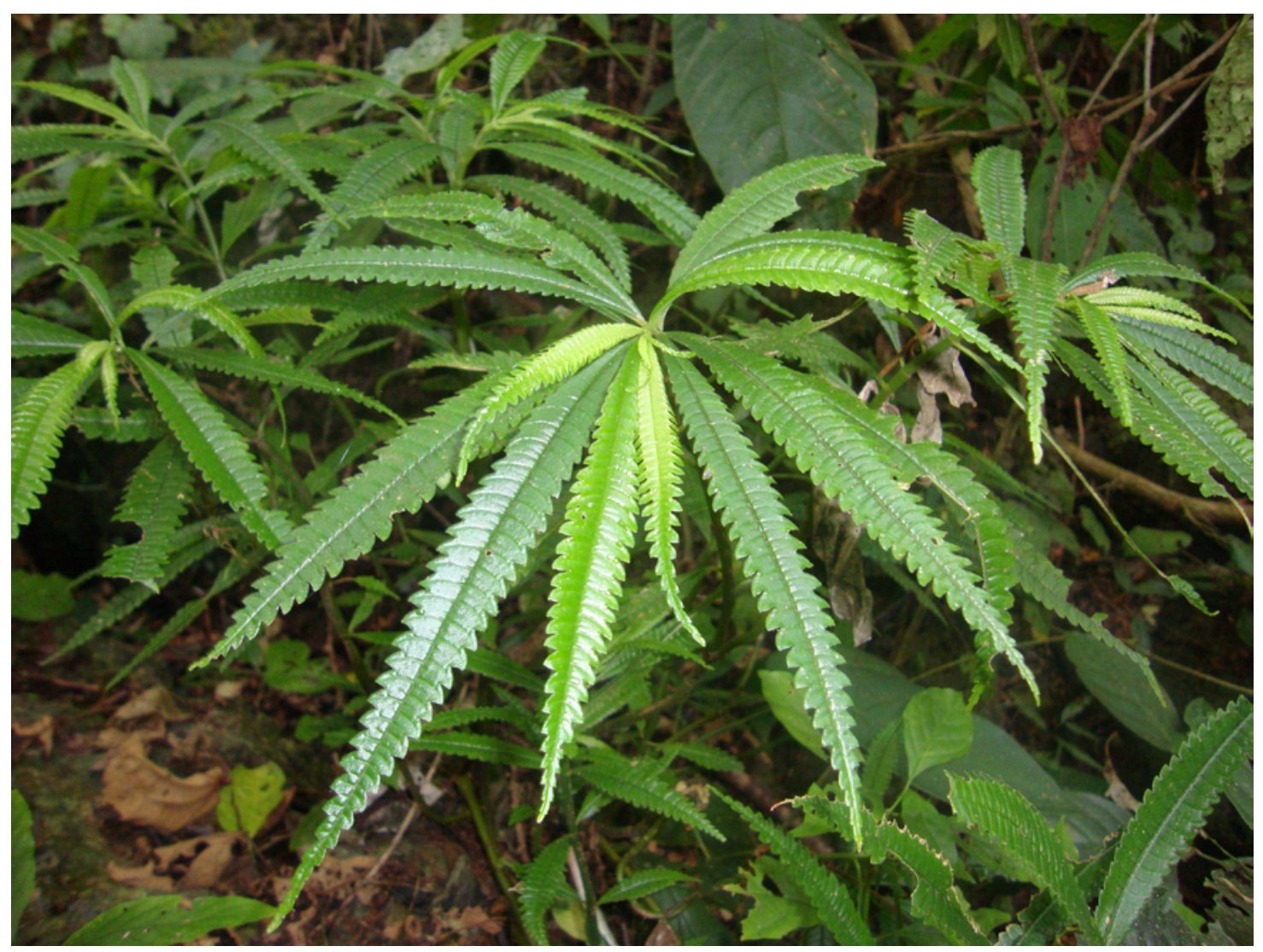

Figura 1. Pilea pteridophylla (A. E Ortíz-Rodríguez 320), en el bosque tropical perennifolio de la zona sujeta a protección ecológica La Pera (Foto Andrés Ernesto Ortíz-Rodríguez) 


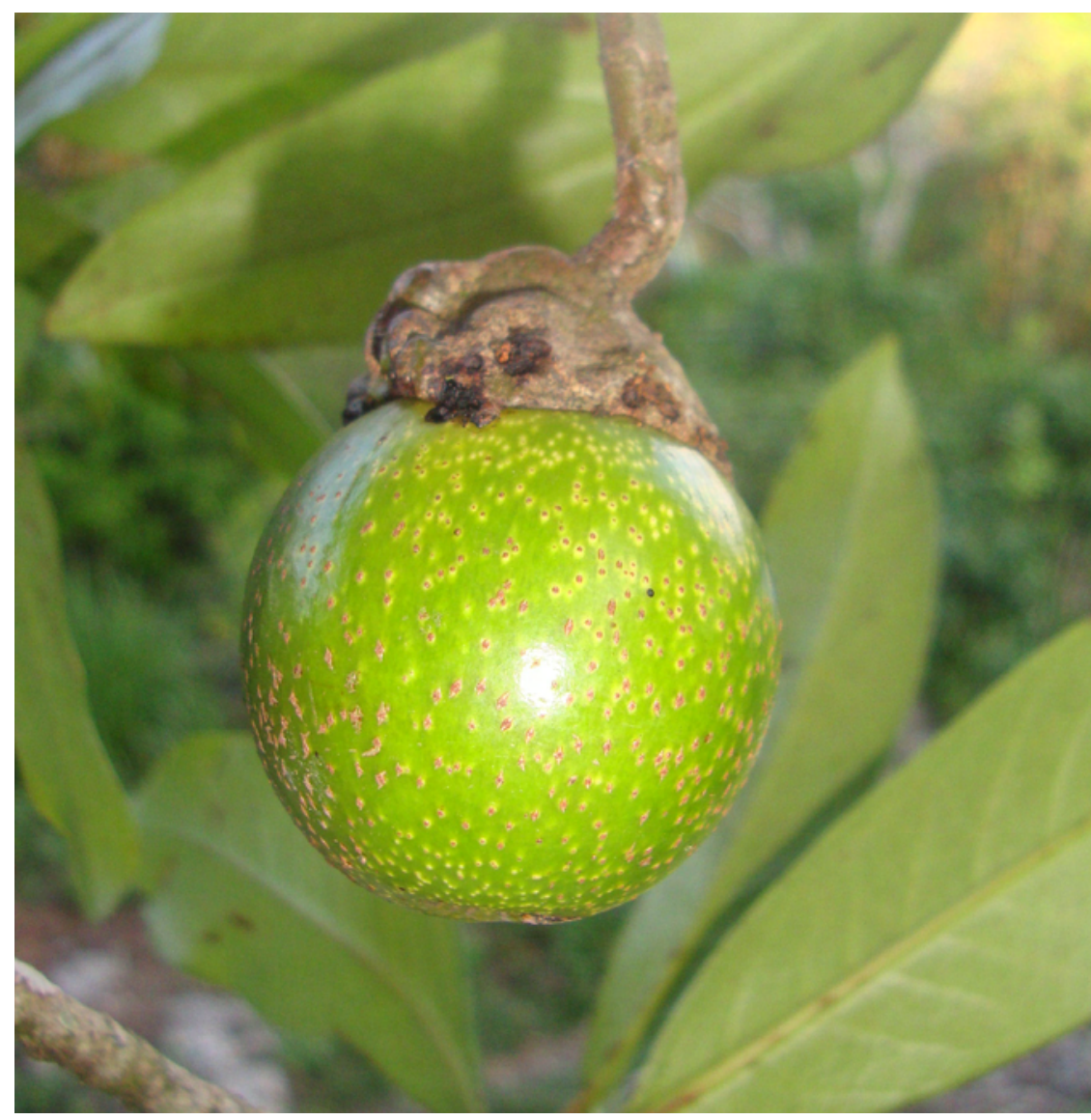

Figura 2. Ocotea heribertoi (A. E Ortíz-Rodríguez 350), fotografía del fruto, en la que se puede observar la forma verrugosa de la cúpula (Foto Andrés Ernesto Ortíz-Rodríguez)

ciaria ligeramente prominente, venas secundarias de 6 a 10. Peciolos de 6 a $14 \mathrm{~mm}$, casi glabros, canaliculados adaxialmente. Inflorescencias de 3 a $10.5 \mathrm{~cm}$, cimoso-paniculadas, esparcidamente adpreso pubescentes, axilares. Flores de 2.5 a $4 \mathrm{~mm}$ de diámetro, verde pálido, perfectas. Tépalos, esparcidamente adpreso pubescente en la superficie externa, más densos en la superficie interna, erectos en la antesis. Estambres 9, 4-loculares, los 6 externos con los filamentos ligeramente más cortos que las anteras, libres, escasamente pubescentes, anteras sin una punta estéril, lóculos en dos filas, apertura introrsa; los 3 internos, con los filamentos casi tan largos como las anteras, escasamente pubescentes, con dos glándulas presentes en la base; estaminodios con una punta engrosada; pistilo de 2.5 a $3 \mathrm{~mm}$, glabro, el estilo casi tan largo como el ovario; receptáculo en forma de copa, glabro por dentro. Fruto poco más de $2.5 \mathrm{~cm}$ de diámetro, globoso, cúpula en forma de tazón, fuertemente verrugosa en la superficie externa, con un sólo margen, los tépalos no persistentes en el fruto.

Ocotea heribertoi, sólo se conocía de dos colecciones del Istmo de Tehuantepec. Esta especie se puede reconocer por sus hojas lisas con un ápice redondeado, por el gran tamaño de los frutos $(4.5 \mathrm{~cm}$ de diámetro en fresco) y las cúpulas grandes y verrucosas. No está claro cuáles son sus relaciones, no hay otras especies en el área que tengan frutos y cúpulas de forma y tamaño similar. En la descripción original (Wendt, 1998), se menciona que la especie presenta en algunas hojas pequeños domacios parecidos a mechones de pelos blancos.
Éstos no se observaron en el ejemplar colectado (Figura 2).

Material examinado: zona sujeta a protección ecológica "La Pera", Berriozábal, Chiapas. Predio "El Zapotal" $16^{\circ} 51^{\prime} 50.6^{\prime \prime} \mathrm{N}$ y $93^{\circ} 19^{\prime} 51.7^{\prime \prime} \mathrm{O}$ 1068 m s.n.m. Ortiz-Rodríguez Andrés Ernesto 350 (HEM).

A pesar de su distribución geográfica tan limitada y la poca frecuencia de sus individuos, Pilea pteridophylla y Ocotea heribertoi, no se encuentran en la NOM-059-SEMARNAT2010 (SEMARNAT, 2010) y aunque en Chiapas se encuentran dentro de la zona sujeta a protección ecológica La Pera, la continuidad de sus poblaciones se ve seriamente amenazada a causa de la deforestación, venta ilegal de flora y extracción de abono, poniendo a ambas especies en riesgo de extinción local.

\section{Agradecimientos}

El primer autor agradece a Héctor Gómez Domínguez y a Yuyini Licona Vera su valiosa ayuda en campo. Agradecemos a Eduardo Estrada y a Rafael Torres Colín los comentarios y sugerencias hechas al documento final.

\section{Literatura citada}

Beutelspacher-Baigts C.R. y Moreno-Molina I. 2010. Nuevos registros y localidades de orquídeas para Chiapas, México. Lacandonia 4:11-15.

Breedlove D.E. 1981. Introducción a la flora de Chiapas. En: López-Sánchez C. Comp. Lecturas Chiapanecas, Tomo VI, pp. 354-356, Gobierno del estado de Chiapas, Tuxtla Gutiérrez.

Contreras-Medina R. y Luna-Vega I. 2001 Presencia de Taxus globosa Schlecht. (Taxaceae) en el estado de Chiapas, México. Polibotánica 12:51-55.

León B. y Martínez-Gordillo M. 2008. Especie nueva de Croton sección Eluteria (Euphorbiaceae) de Chiapas, México. Novon 18:189-191.

López-Velázquez G., Solano-Gómez R. y Pérez-Farrera M.A. 2007. Primer registro de Phloeophila (Orchidaceae: Pleurothallidinae) para la flora de México. Acta Botanica Mexicana 78:77-83. 
Andrés Ernesto Ortiz-Rodríguez, Francisco Lorea Hernández, Miguel A. Pérez-Farrera Alexandre K. Monro.

Lozada-Pérez L. 2008. Especie nueva de Sloanea (Elaeocarpaceae) de Guerrero y Chiapas, México. Revista Mexicana de Biodiversidad 79:303-306.

Martínez-Meléndez J., Pérez-Farrera M.A. y Farrera-Sarmiento O. 2008. Inventario florístico del Cerro El Cebú y zonas adyacentes en la Reserva de la Biosfera El Triunfo (Poligono V), Chiapas, México. Boletín de la Sociedad Botánica de México 82:21-40.

Monro A.K. 1999. Seven new species of Pilea Lindley (Urticaceae) from Mesoamerica. Novon 9:390-400.

Monro A.K. 2004. Three new species and three new names in Pilea (Urticaceae) from New Guinea. Contributions to the flora of Mt Jaya XV. Kew Bulletin 59:573-579.

Monro A.K. 2006. The revision of speciesrich genera: a phylogenetic framework for the strategic revision of Pilea (Urticaceae) based on cpDNA, nrDNA and morphology. American Journal of Botany 93:426-441.

Mullerried F.K.G. 1957. Geología de

Recibido: 14 de octubre de 2011

Aceptado: 21 de diciembre de 2011
Chiapas. Gobierno del estado de Chiapas, Tuxtla Gutiérrez.

Olvera-García M. y Martínez-S E. 2002. Primer registro de Genlisea (Lentibulariaceae) para México. Acta Botanica Mexicana 59:71-73.

Ortega-Ortíz J. y Ortega-Ortíz R. 1998. Una nueva especie de Aristolochia (Aristolochiaceae) de Chiapas, México. Acta Botanica Mexicana 44:1-6.

Ramírez-Marcial N., Ochoa-Gaona S., Gonzáles-Espinosa M. y QuintanaAscencio P.F. 1998. Análisis florístico y sucesional en la estación biológica cerro Huitepec, Chiapas, México. Acta Botanica Mexicana 44:59-85.

Rodríguez A. y Monro A.K. 2008. Cinco nuevas especies de Pilea (Urticaceae) de Costa Rica. Journal of the Botanical Research Institute of Texas 2:995-1007

Rzedowski J. 2006. Vegetación de México. Comisión Nacional para el Cocimiento y Uso de la Biodiversidad, México, D.F. Disponible en linea: <www.biodiversidad.gob.mx/publicaciones/librosDig/pdf/VegetacionMx_Cont.pdf>
SEMARNAT [Secretaría del Medio Ambiente y Recursos Naturales]. 2010. Norma Oficial Mexicana NOM-059SEMARNAT-2010 Protección ambiental - Especies nativas de México de flora y fauna silvestres - Categorías de riesgo y especificaciones para su inclusión, exclusión o cambio.- Lista de especies en riesgo. Diario Oficial de la Federación $2^{\text {a }}$ Sección, 30 de diciembre de 2010.

Van der Werff H. 1991. A key to the genera of Lauraceae in the New World. Annals of the Missouri Botanical Garden 78:377-387.

Van der Werff H. 2002. A synopsis of Ocotea (Lauraceae) in Central America and southern México. Annals of the Missouri Botanical Garden 89:429451.

Villaseñor J.L. 2003. Diversidad y distribución de las Magnolyophyta de México. Interciencia 28:160-167.

Wendt T. 1998. Ocotea heribertoi (Lauraceae), a new species of rain forest canopy tree from the Isthmus of Tehuantepec, Mexico. Lundellia 1:40-45. 